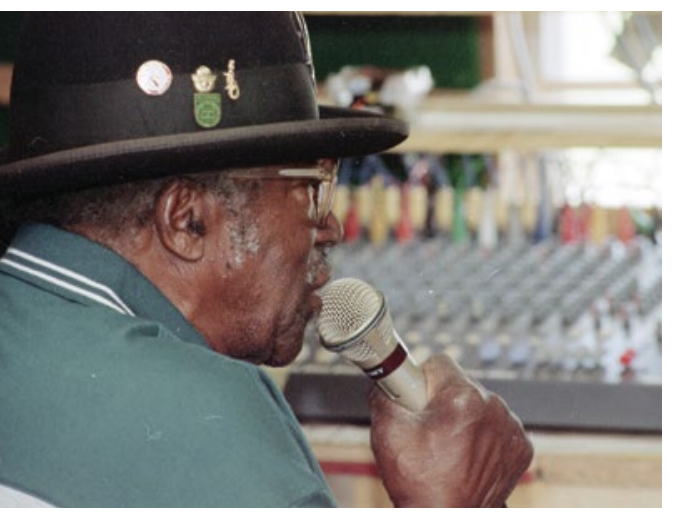

\title{
THE BO DIDDLEY BEAT
}

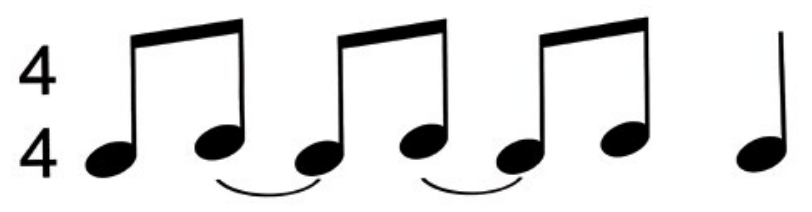

bomp, ba-bomp, ba-bomp, bomp, bomp

Author: Jim Liversidge

Curator of the Popular Culture Collections

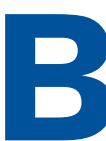
ack in the spring of 2013, the staff of the University of Florida Department of Special and Area Studies Collections hosted a visit by Ellas B. McDaniel (better known by his stage name "Bo Diddley") and his son, Ellas Anthony McDaniel, Jr., in the Grand Reading Room of the Smathers Library. The McDaniels were given a tour of the facilities and collections, but before the afternoon visit was complete the staff was treated to an impromptu performance of anecdotes, poetry, and a few acapella songs, driven by the familiar "Bo Diddley Beat."

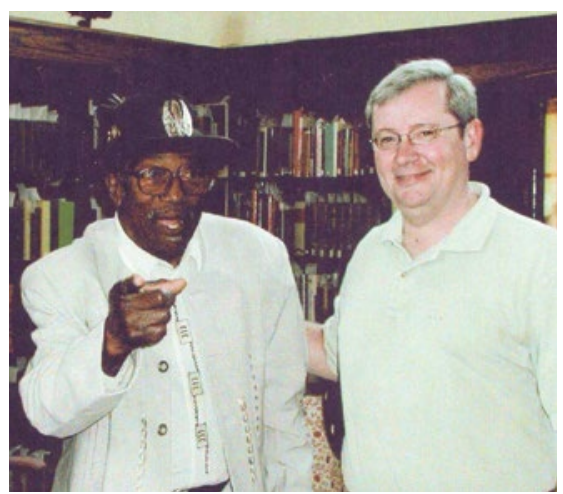

Bo Diddley at the UF Libraries (2003). Photos by Barbara Hood/UF Libraries

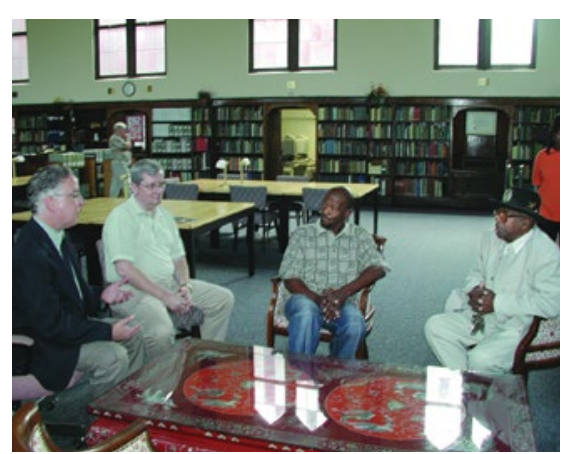

The visit of Rock \& Roll Hall of Famer Bo Diddley was certainly one of the more memorable events in the history of the Grand Reading Room. The memory is still vivid to everyone who had the privilege of speaking with the legendary performer and witnessing his overwhelming desire to entertain, no matter how small the audience. 
Sixteen years later, the legacy of this musical titan is preserved in the Department of Special and Area Studies Collections with his ephemera and artifacts now available to University of Florida students and the general public for research in the Grand Reading Room, where Bo Diddley thrilled a small group of librarians in 2003. THE ELLAS B. McDANIEL (BO DIDDLEY) COLLECTION, acquired in 2017 and 2018, represents the life and career of one of the founders of rock and roll who, in the 1950s, played an important role in the shaping of this new musical genre.

The Ellas B. McDaniel Collection holds an array of items reflecting the musical genius, public performance, and accomplishments of McDaniel, who lived the latter portion of his life in Archer, Florida, near the University of Florida. The collection, established by the George A. Smathers Libraries in collaboration with the Ellas B. McDaniel Irrevocable Trust, ensures the preservation of and access to historical materials of international significance that have particular importance to Floridians.

Bo Diddley brought rhythm and blues elements into the new genre and articulated musical themes that became enduring influences on rock, hip hop, and popular music. His historical significance extends into numerous other areas, and the collection opens avenues for better understanding of technical and performance innovation, international influences in American music, and race and gender in the entertainment industry.

The archival collection is stewarded by
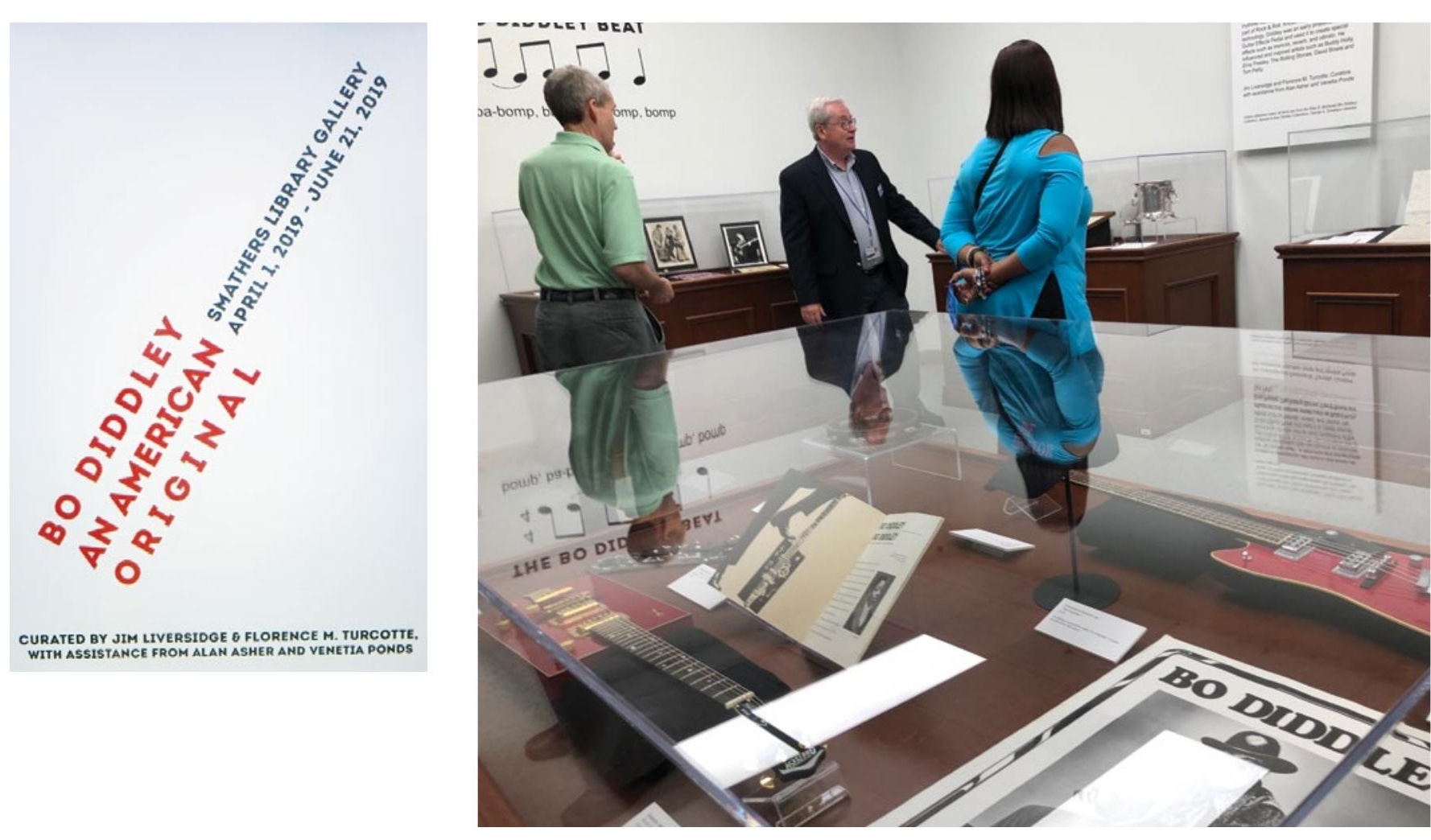
the Special Collections and Area Studies Department of the George A. Smathers Libraries with assistance from library specialists. Library experts in Florida history, music, African American Studies, and popular culture continue to work with conservators of rare materials to ensure preservation and public display of materials in Gainesville and across the United States.

Curators and conservators at the Smathers Libraries identified materials held by the Trust that could be preserved, studied, and exhibited, carefully reviewing and storing materials in boxes and containers designed to ensure long-term preservation of items. While guitars may remain in their original cases at present, conservators separated clothing with tissue paper in boxes made with acid-free paper. Posters have been treated to make them more flexible, with humidifiers adding the right amount of moisture to allow them to be flattened rather than rolled up. Each of the boxes and items selected for the collection under goes a series of evaluations and treatments before being made available to researchers or for exhibitions.

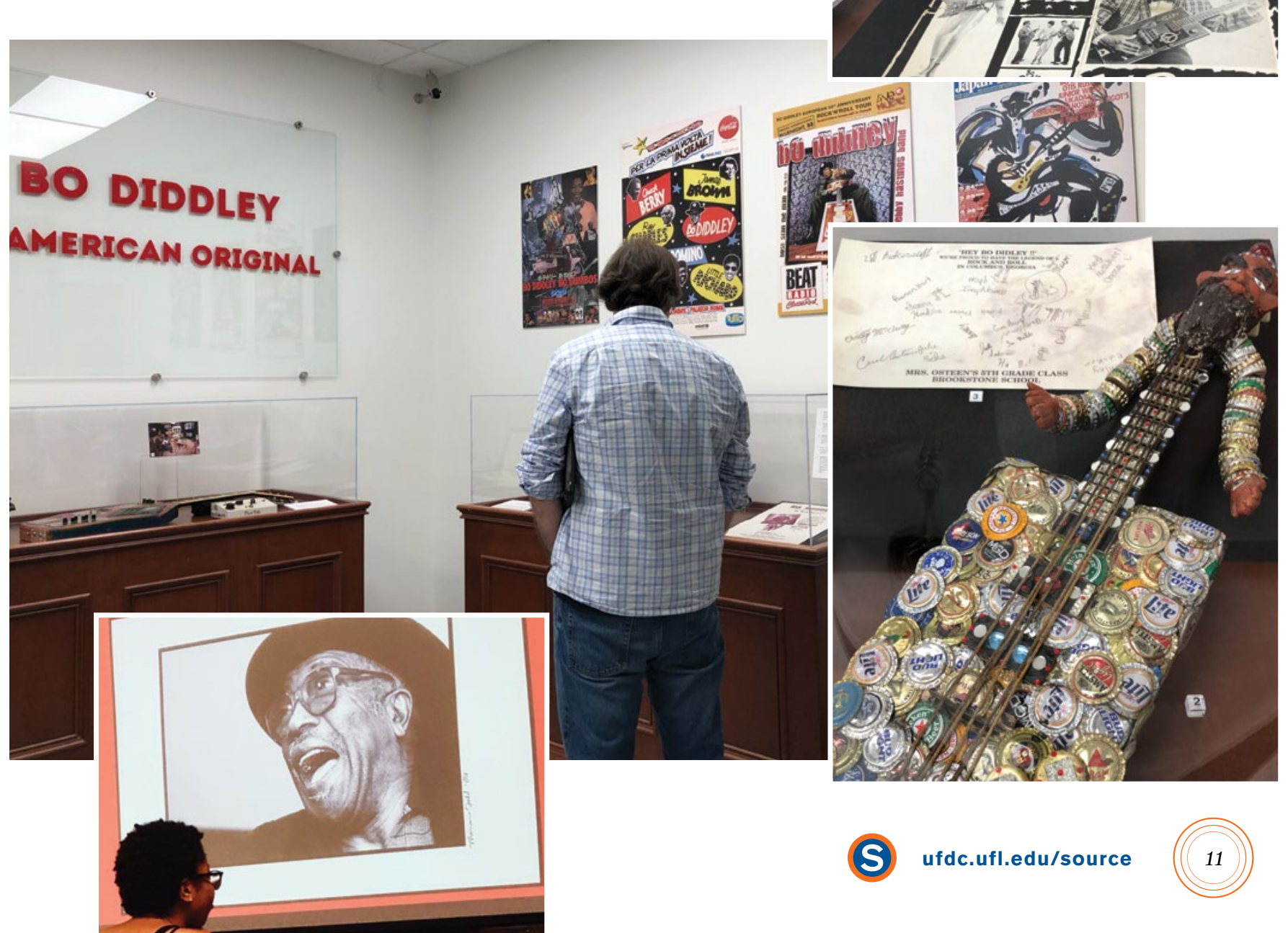




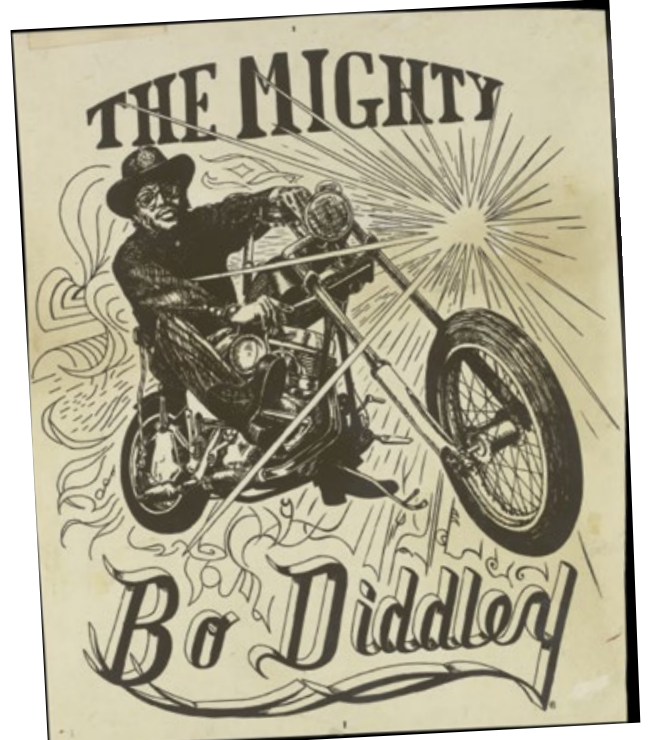

Known as Bo Diddley since his early days as a musician, he was born in Mississippi in 1928. He began his musical career in Chicago, lived in Washington, D.C., and resided in New Mexico and Florida, before passing away in 2008 in Archer, Florida. Placement of the materials at the University of Florida ensures regular public display of an array of historical materials, a high-quality preservation environment, secure use of the collection, and an institutional commitment to the continued promotion of his musical legacy near a community tied closely to McDaniel and his family, as well as to national audiences.

Ellas B. McDaniel's body of work performing as Bo Diddley created a bridge between the genres of rhythm and blues and rock and roll, plucking elements from a variety of musical traditions and transforming them into a signature style. Performers from Elvis Presley to today's hip hop and pop artists adopted and adapted that style for their own music. Throughout a career from the mid-twentieth century to the early twenty-first century, his genius earned him the nick name "The Originator" because of how frequently fellow musicians integrated his innovations into their own styles and performances.

McDaniel also broke barriers by prominently featuring female

"You Don't Know Diddley," Nike Design, Portland, Oregon, 1989

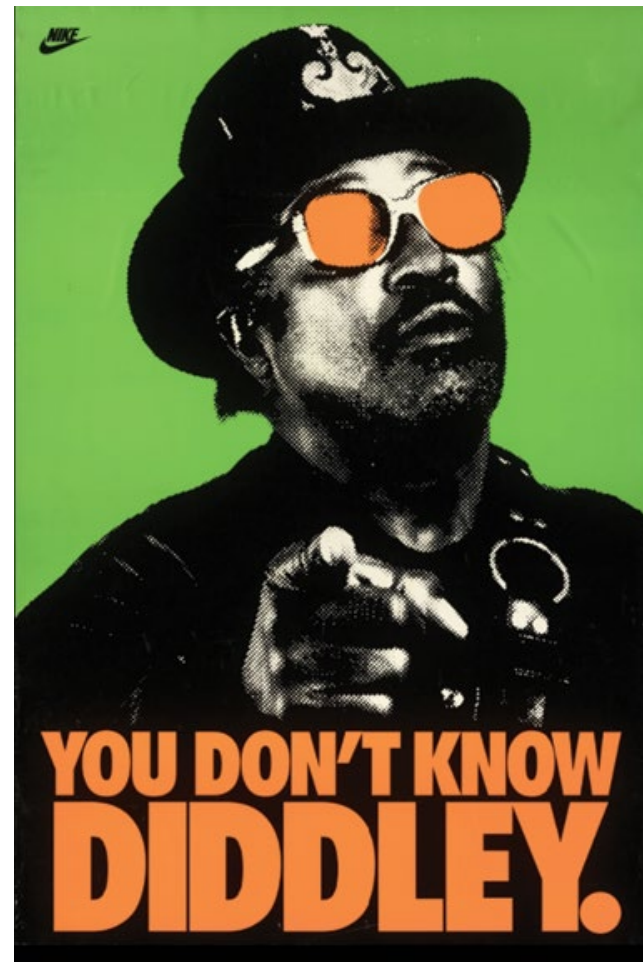
guitarists in his band, unlike any other major male rhythm and blues artist of his day. He struggled to achieve financial success in equal measure to his influence and the popularity of his music. Like too many African American musicians of the twentieth century, early management contracts positioned him unequally in terms of profiting from his own achievements. Elvis Presley and other artists incorporated elements of Bo Diddley's muscular stage movements into their own performances, and others picked up his signature beat.

In 1987, Bo Diddley was inducted into the Rock and Roll Hall of Fame, with a Nike commercial a few years afterward highlighting his ongoing name recognition in popular culture. He continued performing until late in life, earning a Grammy nomination and a Lifetime Achievement Award from the National Academy of Recording Arts and Sciences in the late 1990s, among other awards. The Ellas B. McDaniel (Bo Diddley) Collection enables these important contributions and new perspectives on United States history to be studied and taught at the University of Florida, which awarded McDaniel an honorary doctorate in 2008 (posthumously). 


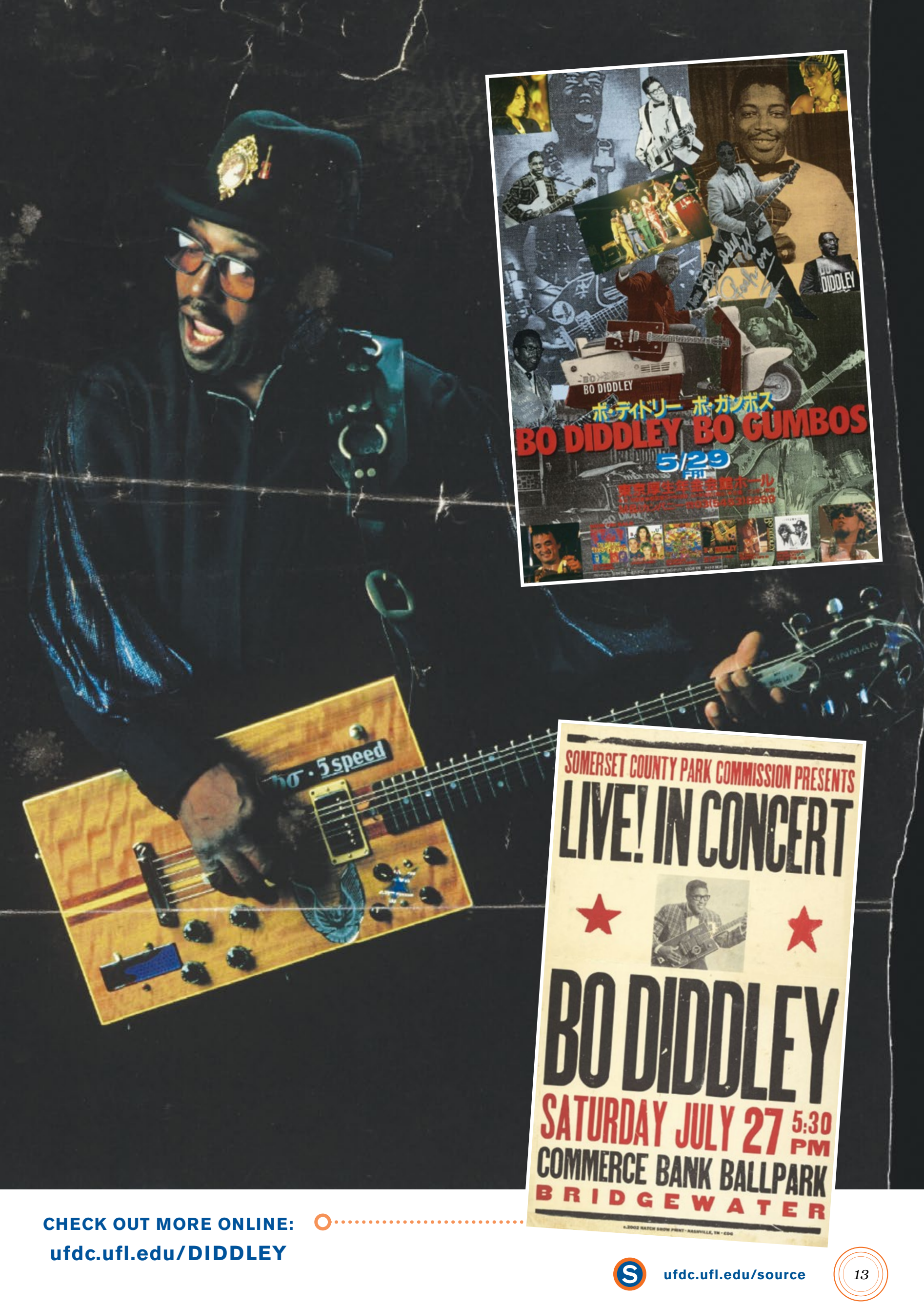

